\title{
Recent results on biomedical problems: A Fourier transform infrared (FT-IR) study
}

\author{
V. Crupi ${ }^{\text {a } *}$, S. Galli ${ }^{\text {a }}$, D. Majolino ${ }^{\text {a }}$, P. Migliardo ${ }^{\text {a }}$, S. Pergolizzi $^{\text {b }}$ and V. Venuti ${ }^{\text {a }}$ \\ ${ }^{a}$ Dipartimento di Fisica dell'Università di Messina \& INFM Sezione di Messina, C.da Papardo S.ta \\ Sperone 31, 98166 Messina, Italy \\ ${ }^{\mathrm{b}}$ Dipartimento di Biomorfologia dell'Università di Messina, C.da Papardo S.ta Sperone 31, 98166 \\ Messina, Italy
}

\begin{abstract}
In the present work, we report on a vibrational study performed on rat encephalon samples and on human tissue affected by cancer, using Fourier Transform Infrared absorbance spectroscopy. As the brain rat tissue is concerned, the FT-IR measurements, performed in the $\mathrm{CH}-\mathrm{OH}$ vibrational stretching region $\left(2400-3800 \mathrm{~cm}^{-1}\right)$, permitted us to reveal the presence of a very diffuse commercial benzodiazepine: VALIUM ${ }^{\circledR}$. The comparison between the spectral features of normal brain and the ones of samples with administrated substance has unambiguously showed that the $\mathrm{CH}$ stretching region seems not to be affected by any change for the pharmacological treatment, instead the $\mathrm{OH}$ band is strongly modified probably due to the presence of a new spectral contribution characteristic of diazepam molecule. In the case of skin tissue the investigation was addressed to characterize the presence of two different pathologies, namely epithelioma and basalioma, and to show clear different spectral features passing from the normal tissue to the malignant one in particular in the region $\left(1500-2000 \mathrm{~cm}^{-1}\right)$ which is typical of the lipids vibrational bands.
\end{abstract}

Keywords: Infrared spectra, medical physics, $\mathrm{OH}$ stretching region

\section{Introduction}

The main components of cells and tissues are given by proteins, nucleic acids, lipids, vitamins and other fundamental supramolecular complexes with highly complicated structures [1-5]. As is well known, passing from the normal tissue to the pathological one, the cellular biochemistry dramatically changes [6,7]. Therefore from a diagnostic and therapeutic point of view, to study the physical and chemical changes occurring in tissues and cells is fundamental [8]. Among the several methods, nowadays applied for investigating medical problems, spectroscopic techniques can provide new and exciting possibilities. In particular Fourier Transform Infrared absorption (FT-IR) spectroscopy $[9,10]$ has become an accepted and common tool in biophysics, providing information concerning the structures and interactions of proteins, lipids, nucleic acids and so on, in isolation and in complex assemblies. It is also true that biochemical information on real-life systems comes from analysis of the above mentioned constituents in their physiological environment, i.e., in cells, tissues and biological fluids and such an information is really difficult to obtain. However if infrared spectroscopy can provide information concerning biochemistry of human cells and tissue, it is logic to think that this technique can be used to study disease processes. In other words infrared spectroscopy is able to detect biochemical changes caused by pathologies, also at very early stage of the disease, due to the fact that any change in the biochemistry

\footnotetext{
*Corresponding author: Dr. Vincenza Crupi, Dipartimento di Fisica, Messina University, P.O. Box 55, 98166 Messina, Italy. Tel.: +39090 6765010; Fax: +39090 395004; E-mail: crupi@ dsme01.unime.it.
} 
tissue must precede any morphological manifestation of the disease in itself. One of the noteworthy advantages in the use of FT-IR spectroscopy is the opportunity to analyse samples, easily prepared, with small size (less than $1 \mathrm{~mm}$ thick and less than $1 \mathrm{~cm}$ in diameter) together with the reliability and the reproducibility of spectral data.

A common problem of recent pharmacological interest is represented by the possibility to reveal substances which are able to get over the haematic-encephalic barrier and hence eventually to recover or damage the brain. The monitoring of drugs distribution by means of traditional and usual methods can result still difficult because of the necessity to use radioactive tracers. In the present paper we deal with a spectroscopic analysis carried out by FT-IR technique on rat brain, performed in the $\mathrm{CH}-\mathrm{OH}$ stretching vibrational region $\left(2400-3800 \mathrm{~cm}^{-1}\right)$ in order to reveal the presence of a very common pharmacological substance (a benzodiazepine) in the brain.

Actually there is a growing belief that passing from normal cell to malignant tumor, the malfunction of protein-encoding genes is involved, and this last can result in a production of proteins in excess or insufficient quantities [11]. Hence the changes in protein or nucleic acid structure between normal and malignant cells can provide valuable clues to understand oncogenesis. Generally in this field the diagnosis is performed by means of invasive techniques based on the removal of a part of the tissue, which can contribute to the progression of the cancer, so the possibility of a non-invasive in vivo measurements of these changes, that could distinguish normal and cancerous tissue, would have really important clinical applications. In the present paper, we also report on a preliminary study on human skin whose goal was essentially to characterize two different kinds of skin cancer by FT-IR response.

\section{Experimental set-up}

\subsection{Rat brain samples}

As the investigation of the presence of the common pharmacological substance, a benzodiazepine, in the rat brain is concerned, we used male Charles River rats, 242-325 g weight that were kept under standard conditions, with free access to food and tap water, for two weeks before treatment. As is well known for good spectroscopic measurements the right sample thickness is essential, hence we obtained thin slides of brain tissue being about $30 \mu \mathrm{m}$ thick, using a standard cutting procedure by a freezer microtome (Reichert Jung), usually followed for routine morphological studies $[12,13]$. So that the obtained slides were laid on $\mathrm{KBr}$ pellets, which were transparent in the analysed wavenumber range $400-4000 \mathrm{~cm}^{-1}$. Thanks to this method we were sure to prevent unwanted contributions in the investigated spectral range, due to the presence of substances eventually used for the fixing procedure. It is worth underlining that the layers were also really homogeneous in the whole extension and this occurrence permitted us to obtain spectra with high reproducibility. The IR measurements were collected both on normal brain slides and on samples with administrated diazepam $\left(\mathrm{C}_{16} \mathrm{H}_{13} \mathrm{ClN}_{2} \mathrm{O}\right)$, in doses consistent with the animals weight and then compared.

\subsection{Skin human tissue}

The studied skin tissue samples were drawn from different parts of human body. The healthy skin specimen came from the leg, a malignant tissue affected, by basalioma, came from the forehead and other malignant samples affected, by epithelioma, from the temporal region. We obtained thin slides of skin tissue, about $20 \mu \mathrm{m}$ thick, by cutting the tissues using the same standard routine followed for the rat 
brain tissue. Also in this case $\mathrm{KBr}$ pellets were used to uphold the skin layers since we were interested in investigating the high wavenumber range $\left(400-4000 \mathrm{~cm}^{-1}\right)$.

For both the two different sets of samples, rat brain and skin tissues, the experimental data were collected by a FT-IR BOMEM DA8 spectrometer which was equipped with a globar lamp as source, a $\mathrm{KBr}$ beamplitter and finally with a DTGS/KBr detector, to work in the above mentioned spectral region. To get a good signal-to-noise ratio and highly reproducible spectra and to avoid any unwanted contribution, we automatically added 100 repetitive scans for each run with a resolution of $4 \mathrm{~cm}^{-1}$, working in dry atmosphere. On the same sample, each measurement has been repeated several times to be sure of the reproducibility of the spectra. A proper baseline has been subtracted from the IR spectra which were successively normalized to take into account the effective number of absorbers. From the well known Lambert-Beer absorbance law, the following expression

$$
\mathrm{ABS}=\log _{10}(\mathrm{REF} / \mathrm{SPC})
$$

in our case hold, where ABS (absorbance) is the spectrum of $\mathrm{KBr}$ matrix together with the sample itself $(\mathrm{KBr}$ pellet + rat brain layer or skin layer) and $\mathrm{REF}$ is the spectrum of the reference matrix $(\mathrm{KBr}$ pellet). To take into account the hygroscopic character of $\mathrm{KBr}$ substrate, we performed absorbance scans on it as well, before and immediately after the measurements on the samples. So that we were certain about the absence of any unwanted wet trace which could be eventually affect the $\mathrm{OH}$ stretching region $3000-3800 \mathrm{~cm}^{-1}$.

\section{Results and discussion}

\subsection{FT-IR measurements on rat brain sample}

We analysed in particular the region ranging from 2800 to $3800 \mathrm{~cm}^{-1}$, typical of $\mathrm{CH}-\mathrm{OH}$ stretching vibrational bands. Obviously IR absorbance measurements were performed on the pure benzodiazepine in powder as well to characterize its main vibrational bands in the region of interest (see inset of Fig. 1). From an inspection of the figure, it appears clear the wide band centred at $3495 \mathrm{~cm}^{-1}$. The IR experimental spectra of rat normal brain tissue and of brain sample treated with the drug, the diazepam, is shown in Fig. 1. As can be seen the lack of the sub-band above mentioned, characteristic of the diazepam, is quite evident in the spectrum of non-treated sample while the $\mathrm{CH}$ stretching band $\left(2800-3000 \mathrm{~cm}^{-1}\right)$ seems not to be modified for the pharmacological treatment, as we actually expected. We analysed the experimental data using a usual best-fit procedure, essentially based on the common criterion that, for a given wide band, the number of sub-bands considered represents the minimum number of contributions and hence the minimum number of reliable parameters, above which the statistical error remains almost constant. From this best-fit procedure, in the case of treated rat brain sample we found the presence of a sub-band centred at $3495.5 \mathrm{~cm}^{-1}$, typical of the vibrational spectrum of diazepam. It has to be noticed that the other sub-bands, in the $\mathrm{OH}$ stretching region of the spectra, refer to molecules containing $\mathrm{OH}$ groups, including water, and that the centre-frequency lays in a spectral region with a great polarizability of the bands widely shifted respect to the free stretching OH groups $\left(\sim 3650 \mathrm{~cm}^{-1}\right)$ [14]. To confirm this evidence, after a right normalization of the absorbance spectra, we subtracted from the spectrum treated with the substance the untreated one (Fig. 2). The resulting spectrum clearly shows a contribution centred at $3495 \mathrm{~cm}^{-1}$, which is absolutely absent in the case of untreated sample.

So that this observation supports the occurrence that the drug, can gets over the haematic-encephalic barrier, spreading in the brain tissue. 


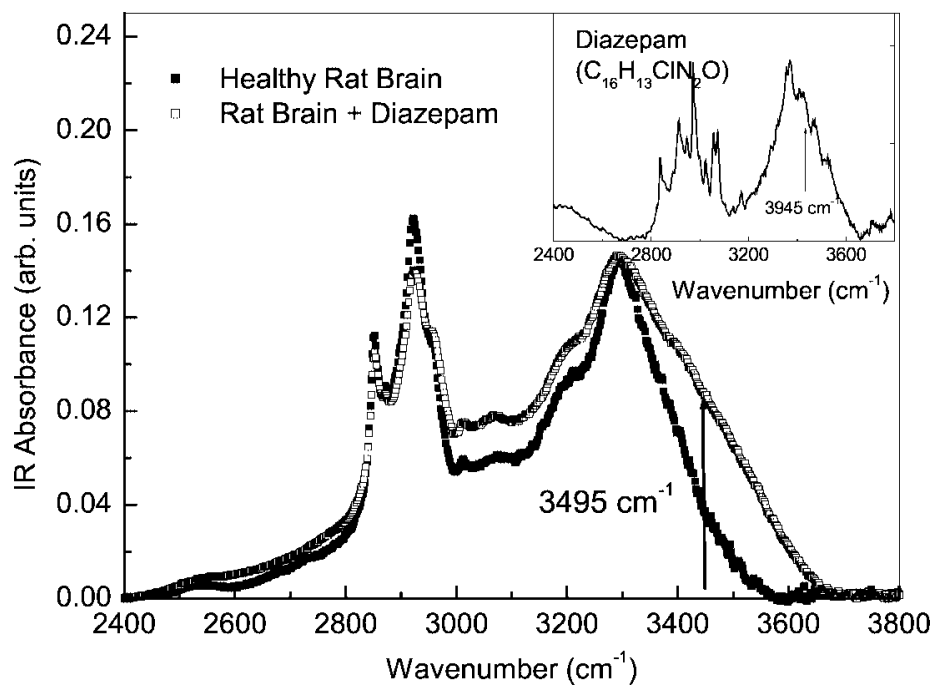

Fig. 1. Experimental IR absorbance spectrum of healthy rat brain (closed squares) and treated with diazepam (open squares). In the inset the IR absorbance spectrum of diazepam is also shown.

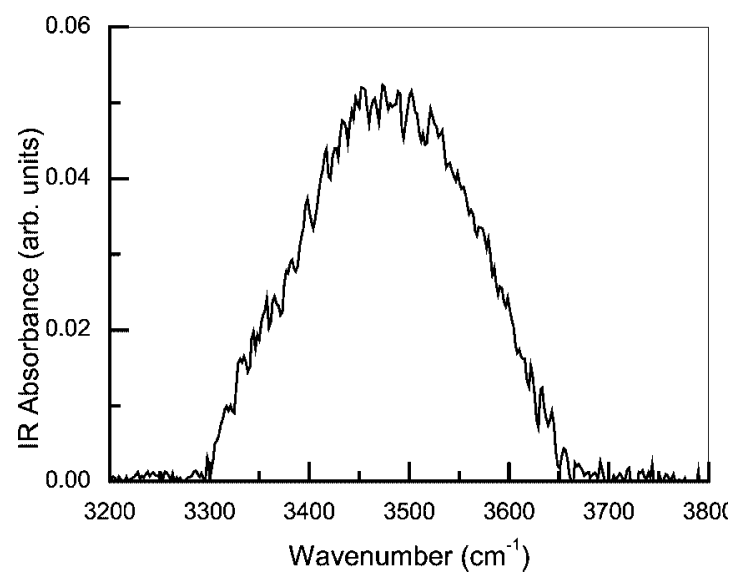

Fig. 2. Spectrum resulting from the subtraction of the untreated spectrum from the treated one.

\subsection{FT-IR measurements on human skin samples}

Skin is essentially composed of three layers: an outer epidermis, an inner dermis and a subcutis [15]. The dermis, 1-3 mm thick, is the important supporting layer. It is a dense irregular connective tissue composed mainly of fibrous collagen, elastic tissue, and ground substances. The collagen fibres are composed of bundles of parallel collagens fibrils, and are oriented in different directions, mostly parallel to the plane of the surface. Dermal collagen fibres contain predominantly type I collagen, whose main component is a fibrous protein and small amounts of type III and type V collagen. So that the derma vibrational features are nearly similar to those type I of collagen. The epidermis is made of epithelial tissue and it is rich of keratinocytes that give the keratin. Essentially its vibrational spectrum is characterized by the typical bands of the proteins. Finally the vibrational spectral profile of subcutis is strictly related to those of lipids with the characteristic band centered at about $1750 \mathrm{~cm}^{-1}$, typical of the $\mathrm{C}=\mathrm{O}$ vibration. 

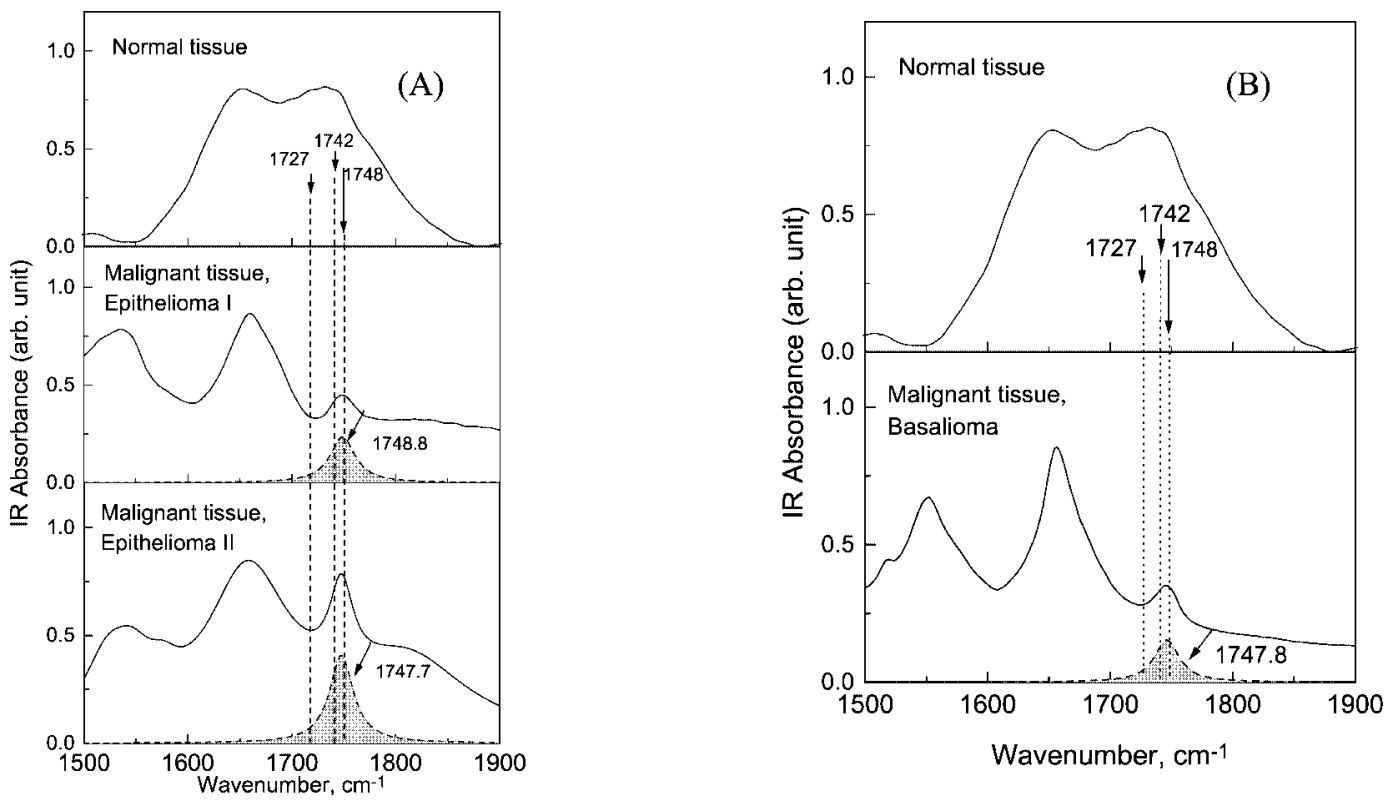

Fig. 3. Experimental IR absorbance spectra of normal and malignant samples affected by epithelioma (A) and basalioma (B), respectively. The dashed arrows indicate the centre frequencies of the main components.

The high complexity of the skin tissue makes the fully characterization quite difficult and a correct bands assignment requires an exact knowledge of the tissue components. This means that, even if the variations observed in the spectra usually reflect changes in the structure due to the presence of a disease like the cancer, one has to take into account other factors as well, such as race, age, food, part of the body that could be alter the amount of lipids, as an example.

In the present work, just due to the complexity of the analysed IR spectra for the reasons above mentioned, even if the IR measurements have been performed in a wide range of wavenumber (400$4000 \mathrm{~cm}^{-1}$ ), we analysed the spectral region from 1500 to $2000 \mathrm{~cm}^{-1}$, which appears particularly involved in the changes caused by the presence of the cancers. The experimental spectra of all the analysed samples were properly normalized and deconvoluted in Lorentzian profiles. We compared the normal samples spectra with the pathological ones. Figure 3(A) shows the IR absorbance spectra of the normal sample and the malignant ones, affected by epithelioma. The main changes observed can be attributed to the sub-bands, centred at $1727 \mathrm{~cm}^{-1}$ and $1742 \mathrm{~cm}^{-1}$ that are the $\mathrm{C}=\mathrm{O}$ vibration of cellular membranes lipids, and at $1748 \mathrm{~cm}^{-1}$ that is the $\mathrm{C}=\mathrm{O}$ vibration of phospho-lipids, which are present only in the healthy sample. As can be seen from an inspection of Fig. 3, in the pathological samples only the band connected with phospho-lipids survives. The same observation can be made in the case of tissue affected by basalioma (see Fig. 3(B)).

\section{Conclusions}

We performed a Fourier Transform Infrared (FT-IR) analysis on rat encephalon samples, in the $\mathrm{CH}-\mathrm{OH}$ vibrational stretching region $2400-3800 \mathrm{~cm}^{-1}$ and on human skin tissue in the region $1500-2000 \mathrm{~cm}^{-1}$. Our work was essentially addressed to show that the FT-IR absorption technique provides reliable, re- 
producible spectra which can be used to unambiguously distinguish normal from treated and/or injured specimens.

In the case of the rat brain samples, the aim of the spectroscopic study was addressed to reveal if a drug substance, in our case a commercial benzodiazepine, was able, or not, to get over the haematicencephalic barrier. It was found that in analysed spectral range, the $\mathrm{OH}$ stretching band for the sample treated pharmacologically is strongly modified due to the presence of a contribution which is characteristic of the diazepam molecule. In the case of the investigation of normal and malignant skin samples the infrared spectroscopy has demonstrated to be a powerful tool to study disease processes.

It is worth emphasizing that, compared with other analytical experimental techniques, FT-IR spectroscopy has the advantage of fast characterization, good reproducibility, and the possibility of examining unfixed samples with reduced dimensions.

\section{References}

[1] M. Jackson and H.H. Mantsch, FTIR spectroscopy in the clinical sciences, in: Advances in Infrared and Raman Spectroscopy, R.J.H. Clark and R.E. Hester, eds, Wiley Hayden, 1996, pp. 185-215.

[2] M. Jackson and H.H. Mantsch, CRC Crit, Rev. Biochem. Mol. Biol. 30 (1995), 95.

[3] H.L. Casal and H.H. Mantsch, Biochim. Biophys. Acta 779 (8) (1994), 381.

[4] M. Mathlouthi and J.L. Koenig, Adv. Carbohydr. Chem. Biochem. 44 (1986), 7.

[5] E. Taillandier, J. Liquier and J.A. Taboury, in: Advances in Infrared and Raman Spectroscopy, R.J.H. Clark and R.E. Hester, eds, Wiley, New York, 1985, pp. 65-80.

[6] I. Nabiev, I. Chourpa and M. Manfait, J. Raman Spectrosc. 25 (1994), 13.

[7] P.T.T. Wong and B. Rigas, Appl. Spectrosc. 44 (1990), 1715.

[8] D.M. Haaland, H.D.T. Jones and E.V. Thomas, Appl. Spectrosc. 51 (1997), 340.

[9] P. T.T. Wong, K. Wong and M.F.K. Fung, Appl. Spectrosc. 47 (1993), 1058.

[10] V. Crupi, D. Majolino, P. Migliardo, M.R. Mondello, M.P. Germanò and S. Pergolizzi, Vibrat. Spectrosc. 25 (2001), 213.

[11] S.H. Hawi, W. Campbell, A. Kajdacsy-Balla, R. Murphy, F. Adar and K. Nithipatikom, Cancer Lett. 110 (1996), 35.

[12] B. Rigas, S. Murgello, I.S. Goldman and P.T.T. Wong, Proc. Natl. Acad. Sci. USA 87 (1990), 8140.

[13] H.J. Kim, J.V. Bruckener, C.E. Dallas and J.M. Gallo, Toxicol. Appl. Pharmacol. 102 (1990), 50.

[14] I. Olovsson and P.G. Jonsson, in: The Hydrogen Bond, P. Shuster, G. Zundel and C. Sandorfy, eds, North-Holland, Amsterdam, 1976, pp. 393-495.

[15] S. Vaezy, L. Smith, A. Milaninia, J.I. Clark, J. Electron Microsc. 44 (1995), 35. 


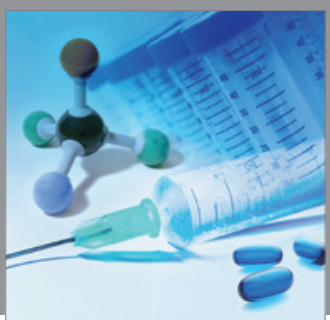

International Journal of

Medicinal Chemistry

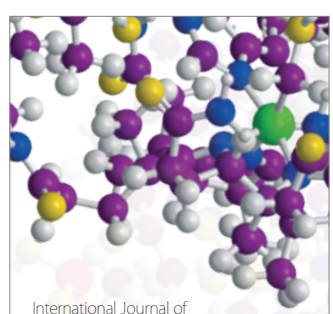

Carbohydrate Chemistry

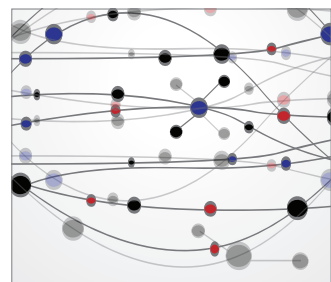

The Scientific World Journal
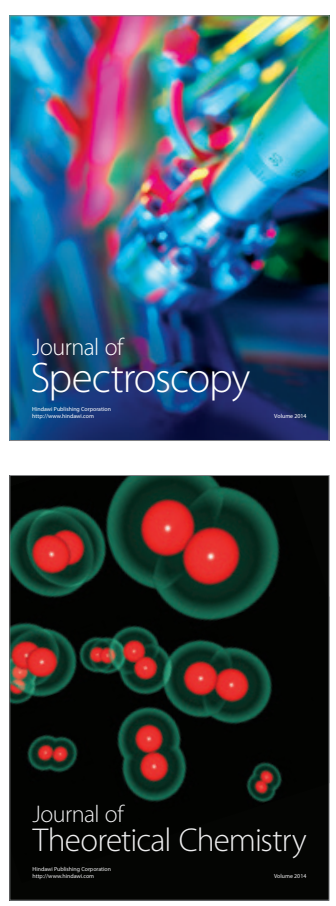
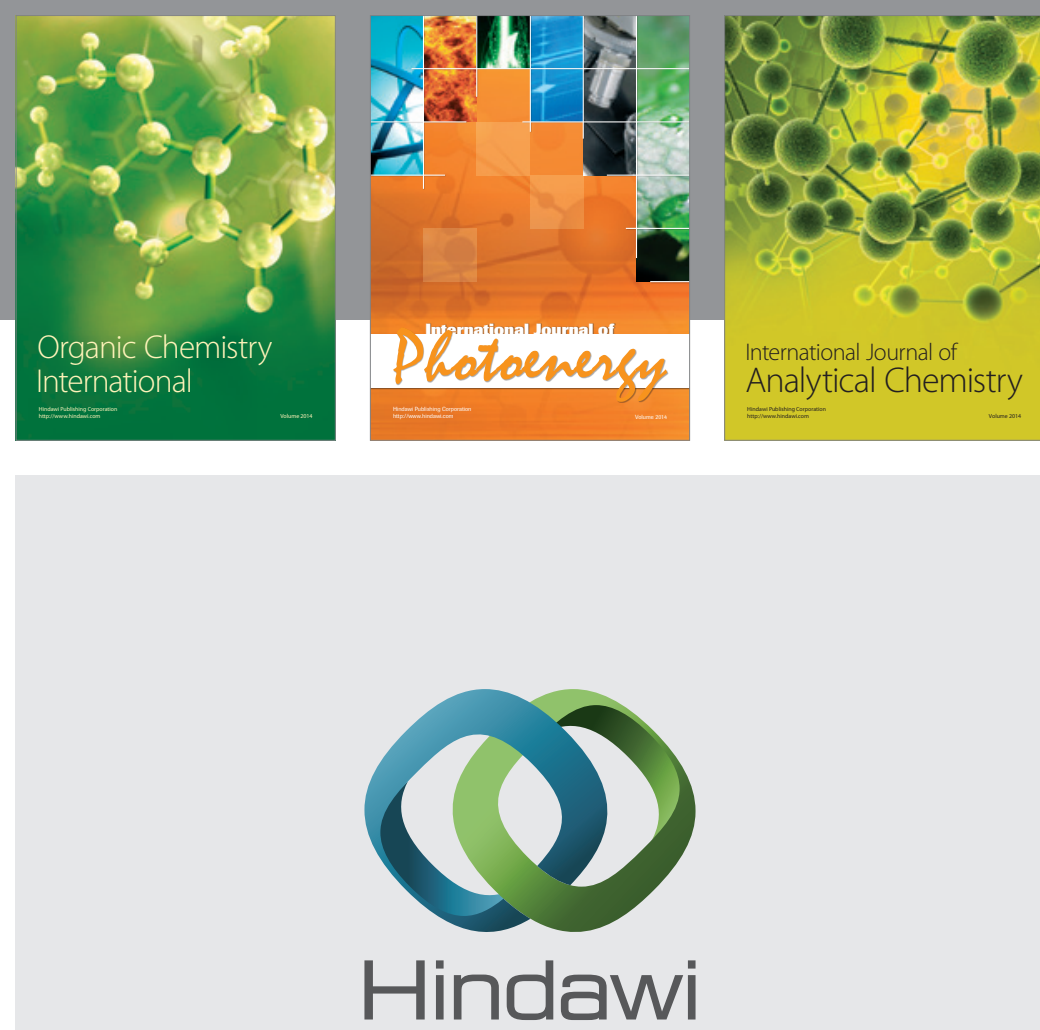

Submit your manuscripts at

http://www.hindawi.com
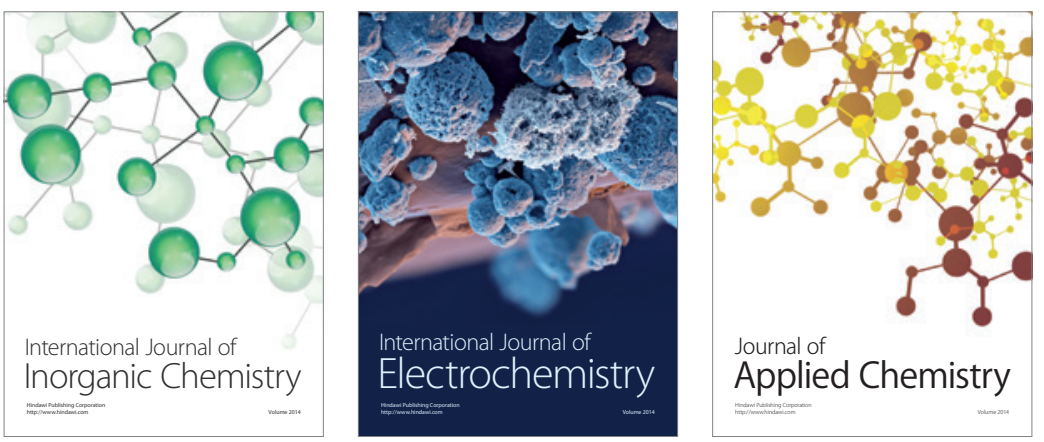

Journal of

Applied Chemistry
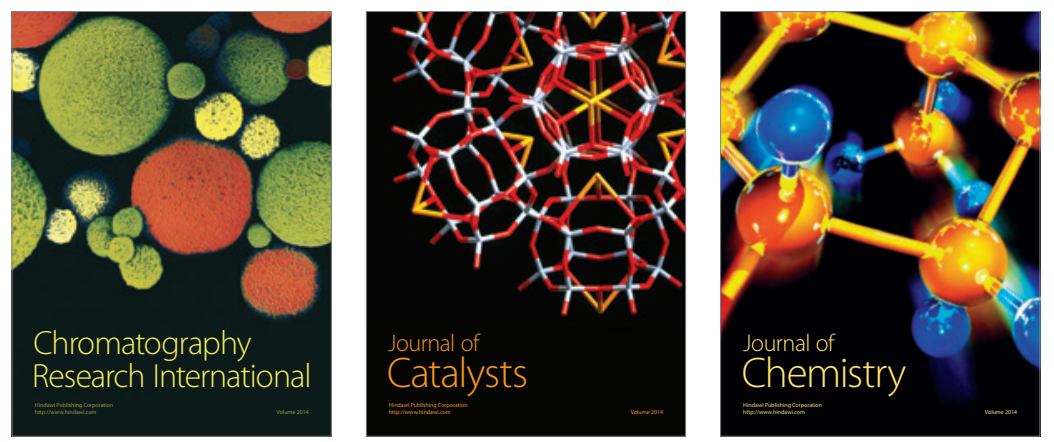
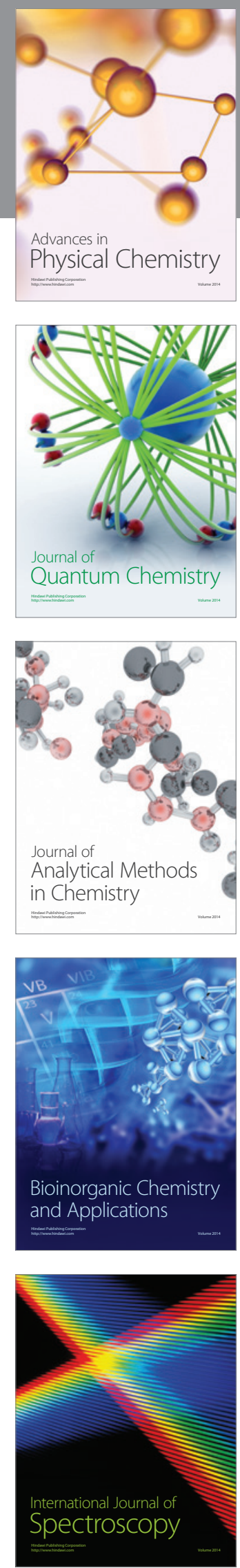